\title{
Explosive Synchronization in Complex Dynamical Networks Coupled with Chaotic Systems
}

\author{
Juan Chen ${ }^{*}$, Hao Tu${ }^{1}$, Junchan Zhao ${ }^{2}$ \\ ${ }^{1}$ College of Science, Wuhan University of Science and Technology, Wuhan, China \\ ${ }^{2}$ School of Mathematics and Statistics, Hunan University of Technology and Business, Hunan, China \\ Email: *jc1204@126.com
}

How to cite this paper: Chen, J., Tu, H. and Zhao, J.C. (2019) Explosive Synchronization in Complex Dynamical Networks Coupled with Chaotic Systems. World Journal of Mechanics, 9, 245-258.

https://doi.org/10.4236/wjm.2019.911016

Received: October 5, 2019

Accepted: November 5, 2019

Published: November 8, 2019

Copyright $\odot 2019$ by author(s) and Scientific Research Publishing Inc. This work is licensed under the Creative Commons Attribution International License (CC BY 4.0).

http://creativecommons.org/licenses/by/4.0/

\section{c) (i) Open Access}

\begin{abstract}
Explosive synchronization (ES), as one kind of abrupt dynamical transitions in nonlinearly coupled systems, has become a hot spot of modern complex networks. At present, many results of ES are based on the networked Kuramoto oscillators and little attention has been paid to the influence of chaotic dynamics on synchronization transitions. Here, the unified chaotic systems (Lorenz, Lü and Chen) and Rössler systems are studied to report evidence of an explosive synchronization of chaotic systems with different topological network structures. The results show that ES is clearly observed in coupled Lorenz systems. However, the continuous transitions take place in the coupled Chen and Lü systems, even though a big shock exits during the synchronization process. In addition, the coupled Rössler systems will keep synchronous once the entire network is completely synchronized, although the coupling strength is reduced. Finally, we give some explanations from the dynamical features of the unified chaotic systems and the periodic orbit of the Rössler systems.
\end{abstract}

\section{Keywords}

Complex Networks, Explosive Synchronization, Chaotic Systems

\section{Introduction}

Complex networks are ubiquitous in the world, such as transportation networks, Internet, wireless networks and phone networks. In 1998, Watts and Strogatz presented the small-world network model [1] [2], which was a network model with the small average path length but the large clustering coefficient. Later, the 
scale-free network model whose degree distribution obeys a power-law distribution [3], is proposed by Barabási and Albert in 1999. Since then, they make great progress of complex networks and become the theoretical basis of modern complex networks. Synchronization, as a collective dynamical behavior, is an important and interesting direction of complex networks. In the past two decades, synchronization of complex networks has extensively attracted increasing attention and practical applications [4]-[10], such as parallel computing. However, all the reported cases examples are of continuous phase transitions.

Recently, it has been shown that discontinuous transitions can take place in networks of periodic oscillators [11], called explosive synchronization (ES). As one kind of abrupt dynamical transitions in nonlinearly coupled systems, it has attracted widespread attention from the systems science community [12]-[17]. Traditionally, the master stability function is used to study the continuous change of systems and to focus on the synchronizability of networks, rather than synchronization processes. However, explosive synchronization is commonly observed in heterogeneous networks. Gómez-Gardeñes et al. [11] proposed that ES could occur in the networked Kuramoto oscillators and the following two conditions are satisfied: 1) a scale-free network structure and 2) the existence of a positive correlation between the natural frequency of an oscillator and its degree. There are many systems in the world that are not Kuramoto systems, but chaotic systems. A large system is said to undergo a phase transition when one or more of its properties change abruptly after a slight change in a controlling variable. If the transitions are discontinuous or abrupt, they are called a first-order. Conversely, when the transitions are continuous or smooth, they are second-order. Generally, there are two main factors to influence phase transitions of complex networks as following: 1) the topological structure of the network and 2) the dynamics of the system. More recently, there are many studies of ES based on Kuramoto and little attention is paid to other chaotic dynamics in [13] [14] [15]. Zhao [12] studied explosive synchronization of complex networks with different chaotic oscillators and indicated that explosive synchronization only takes place in the coupled Lorenz systems. However, Zhao only considers the process from incoherence to synchrony, ignoring the process from synchrony to incoherence. Generally speaking, explosive synchronization can be said to happen in complex networks when the following conditions are satisfied: 1) the emergence of the first-order transition and 2) the hysteresis curve appears in the process from synchrony to incoherence.

Motivated by the above discussions, this paper investigates explosive synchronization in complex dynamical networks coupled with chaotic systems. The chaotic systems to be studied contain the unified chaotic systems (Lorenz, Lü and Chen) [18] and the Rössler systems [19]. The advantage of the unified chaotic system is that it can be adjusted to different chaotic systems by changing one parameter. Compared with the Kuramoto model, the chaotic systems are used more widely. In addition, the networks are constructed in [20] that generate a 
one-parameter family of complex networks. It can easily adjust the topological structures of networks and measure the degree of heterogeneity of the final networks. The numerical simulations show that explosive synchronization is obviously discovered in the coupled Lorenz systems of different structures, but there are not obvious first-order transitions and hysteresis curves for coupled Lü and Chen systems. Moreover, for the coupled Lü and Chen systems there are some shocks in synchronization transitions. Additionally, for the coupled Rössler systems, it has a surprising phenomenon that it cannot desynchronize once it is synchronized. Our researches consider not only the dynamics of chaotic systems, but also the network structures.

The rest of this paper is organized as follows. Section 2 introduces the network model and preliminaries. Sections 3 presents lots of simulations of the coupled unified chaotic systems and analyzes the results from the dynamical behaviors of systems. Section 4 provides some simulations of coupled Rössler systems and discusses the phenomenon from the view of the periodic orbit. Finally, Section 5 gives the conclusion of this paper.

\section{Model Description and Preliminaries}

Consider an undirected and unweighted network of $N$ coupled chaotic oscillators as follows:

$$
\dot{x}_{i}=F\left(x_{i}\right)+\lambda \sum_{j=1}^{N} c_{i j} x_{j},
$$

where $i=1,2,3, \cdots, N, \quad x_{i} \in R^{m}$ is the $m$-dimensional state variable of the node $i$ and $F(\cdot): R^{m} \rightarrow R^{m}$ is a continuous vector function. The outer-coupling matrix $\left(c_{i j}\right)_{N \times N}$ is defined as $C=A-D$ and describes the coupling topology of the network, where $A=\left(a_{i j}\right)_{N \times N}$ is the adjacency matrix of the network ( $a_{i j}=1$ if nodes $i$ and $j$ are connected, and 0 otherwise), $D=\left(d_{i j}\right)$ is the diagonal matrix with $d_{i i}=\sum_{j=1}^{N} a_{i j}$ and $\lambda$ is the coupling strength.

Since the inner coupling matrix is the identity matrix, the synchronization region is unbounded based on the master-stability-function approach [21]. When the coupling strength becomes large enough, the complex network (1) will achieve complete synchronization. The network (1) is said to achieve global complete synchronization if $\lim _{t \rightarrow \infty}\left\|x_{i}(t)-x_{j}(t)\right\|=0$, for $i, j=1,2, \cdots, N$. To monitor such synchronization transition as $\lambda$ grows, the global order parameter $p(t)$ and the local error parameter $e_{i}(t)$ are defined.

The global order parameter $p(t)$ is described in [12]:

$$
p(t)=\frac{2}{N(N-1)} \sum_{j=1}^{N-1} \sum_{i=j+1}^{N} \theta\left(r-\left\|x_{i}(t)-x_{j}(t)\right\|_{2}\right),
$$

where $\theta$ is Heaviside function

$$
\theta(x)=\left\{\begin{array}{l}
0, x \leq 0 \\
1, x \geq 1
\end{array}\right.
$$

and $r$ is a small positive constant. Obviously, $p(t) \in[0,1]$, can quantify the de- 
gree of synchronization among the $N$ oscillators and measure the coherence of the collective motion. The network (1) is fully synchronized when the value $p=1$, while the network (1) is the incoherent state when the value $p=0$.

The local error parameter is described as

$$
e_{i}(t)=\left\|x_{i}-\bar{x}\right\|,
$$

where $i=1,2, \cdots, N$ and $\bar{x}=\sum_{i=1}^{N} x_{i} / N$. The $e_{i}(t)$ reflects the motion state of each node in some extent and can measure whether the $i$-th node is synchronized. The network (1) is fully synchronized when the value $e_{i}(t)=0$ for all nodes $i=1,2, \cdots, N$.

In this paper, the unified chaotic systems (Lorenz, Lü, Chen) and Rössler systems are selected to study explosive synchronization of coupled chaotic systems in different network structures.

The unified chaotic system is described by [18]:

$$
\left\{\begin{array}{l}
\dot{x}=(25 \alpha+10)(y-x) \\
\dot{y}=(28-35 \alpha) x-x z+(29 \alpha-1) y \\
\dot{z}=x y-\frac{1}{3}(\alpha+8) z
\end{array}\right.
$$

where $\alpha$ is a parameter. The system is chaotic when the parameter $\alpha \in[0,1]$. The unified chaotic system is essentially the convex combinations of Lorenz system and Chen system. It represents the whole family of infinitely many chaotic systems in the middle, while the Lorenz system and the Chen system are just two extreme cases. According to the value of parameter, the system (5) can be classified as follows: when $0 \leq \alpha<0.8$, the system (5) belongs to the generalized Lorenz system; when $\alpha=0.8$, the system (5) belongs to the generalized Lü system; when $0.8<\alpha \leq 1$, the system (5) belongs to the generalized Chen system.

The Rössler system is described by [19]:

$$
\left\{\begin{array}{l}
\dot{x}=-(y+z) \\
\dot{y}=x+a y \\
\dot{z}=b+z(x-c)
\end{array},\right.
$$

where $a=0.15, b=0.2, c=5.7$.

In further exploring the influence of topological structures on explosive synchronization, we use a one-parameter family of complex networks in [20]. This parameter measures the degree of heterogeneity of the final networks by interpolating from Barabási-Albert (BA) to Erdös-Rényi (ER) networks by tuning $\beta \in[0,1]$. Let us assume the final size of the network to be $N$. The network is generated starting from a fully connected core of $m_{0}$ nodes and a set $u(0)$ of $N-m_{0}$ unconnected nodes. Then, at each time step, a new node (not selected before) is chosen from $u(0)$ and linked to $m$ other nodes. Each of the $m$ edges is linked with probability $\beta$ to a randomly chosen node (avoiding self-connections) from the whole set of $N-1$ remaining nodes and with probability $(1-\beta)$ following a linear preferential attachment strategy [20]. Finally, the process is re- 
peated $N-m_{0}$ time steps. With this procedure, the networks interpolating between the limiting cases of $\mathrm{BA}(\beta=0)$ and $\mathrm{ER}(\beta=1)$ structure are generated.

\section{The Unified Chaotic System as Node Dynamics}

In this section, a lot of numerical simulations of the unified chaotic systems (Lorenz, Lü, and Chen) are presented in different network structures. Figure 1 $(\alpha=0)$, Figure $2(\alpha=0.8)$ and Figure $3(\alpha=1)$ respectively show synchronization diagrams of Lorenz, Lü and Chen systems with different network structures. For each panel in them, the synchronization values $p(t)$ are computed, labeled as Forward and Backward continuations. The red lines represent Forward and the yellow lines represent Backward. In Forward numerical trials, the state of the network is monitored as a function of the coupling strength, by gradually increasing the value of $\lambda$ in steps $\delta \lambda$ from $\lambda_{0}$. Therefore, the value of the global order parameter $p$ is computed for $\lambda_{0}, \lambda_{0}+\delta \lambda, \cdots, \lambda_{0}+n \delta \lambda$. Furthermore, we look for a first-order transition and a synchronization hysteresis, so we do the simulations also in the reverse way, i.e., starting from $\lambda_{0}+n \delta \lambda$, and gradually decreasing the coupling strength by $\delta \lambda$ at each step. Correspondingly, Figure $4(\alpha=0)$, Figure $5(\alpha=0.8)$ and Figure $6(\alpha=1)$ respectively show the local error diagrams for coupled Lorenz, Lü, and Chen systems with different network structures under Forward continuations. Here, we select $\delta \lambda=0.01$ and set $r=0.01$ in the Heaviside function.

For each panel in Figure 1, we can find that for the coupled Lorenz systems, a sharp, first-order synchronization transition appears from the incoherent state to full synchronization. The global order parameter remains $p \simeq 0$ until the network begins to synchronize in which $p$ jumps suddenly to $p \simeq 1$ during Forward continuations. This result illustrates that all nodes of the network have achieved the synchronization state in an instant when the coupling strength increases to a threshold $\lambda_{f}$. Moreover, the Backward continuation also shows an abrupt transition from the fully synchronized state to the incoherent one. Similarly, this result also illustrates that all nodes of the network fall into the incoherent state in an instant when the coupling strength decreases to a threshold $\lambda_{b}$. However, the two abrupt phase transitions happen at different threshold i.e. $\lambda_{f} \neq \lambda_{b}$ so that the whole synchronization diagram displays a strong hysteresis seen in yellow lines and meanwhile indicates that explosive synchronization is irreversible. In addition, the order parameter $p$ has a slight oscillation before achieving complete synchronization. In order to further explore the change of the dynamical behavior of each node, the corresponding local error diagrams are plotted along the forward continuation and it can monitor the evolution of the dynamics for every node defined in section 2, as shown in Figure 4. $e_{i}(\lambda)=0$ represents that the entire network is in a synchronous state, and the simultaneously sharp drop indicates the emergence of explosive synchronization. We can see that dynamical behaviors of nodes retain, i.e., $e_{i} \neq 0$ until the coupling strength $\lambda=\lambda_{f}$, which indicates the abrupt synchronization observed in Figure 1. 


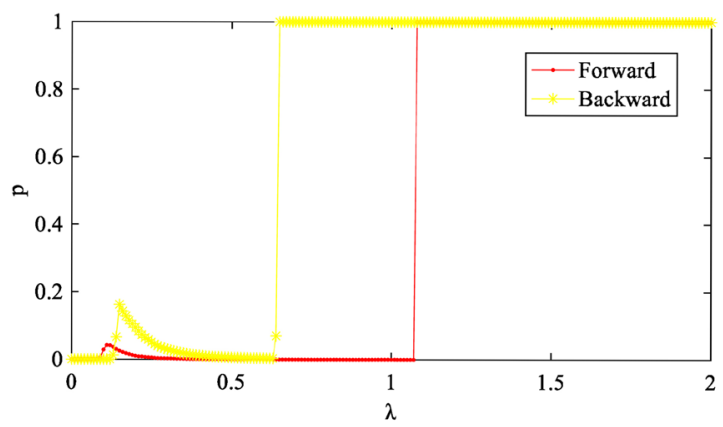

(a)

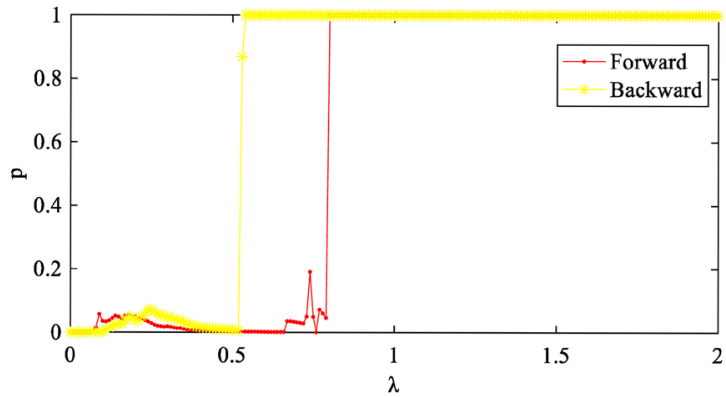

(c)

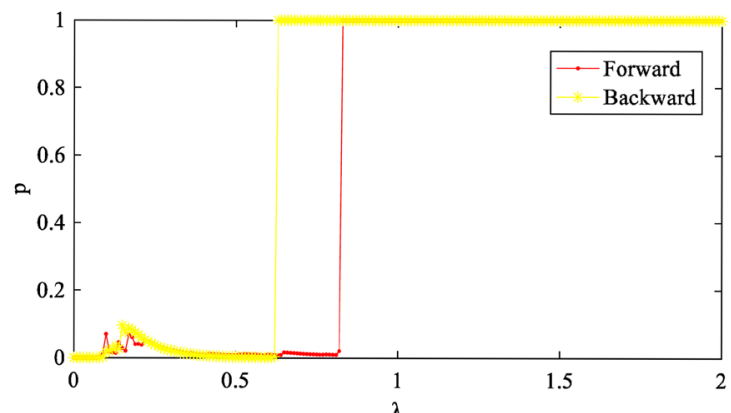

(b)

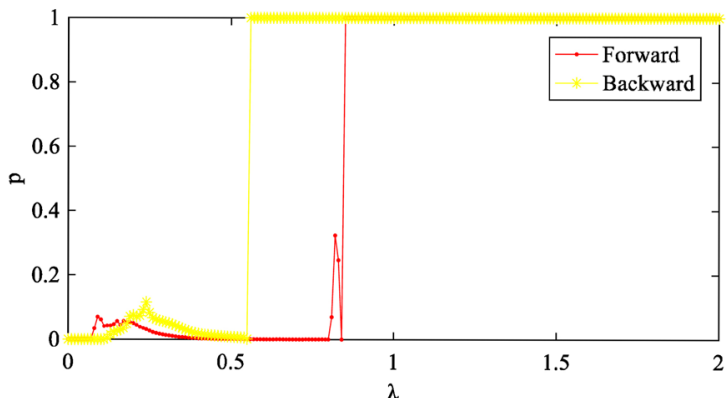

(d)

Figure 1. (color online) The synchronization diagrams $p(\lambda)$ of the networked Lorenz systems for different networks constructed using the model introduced in [20]. The $\beta$ values in each panel are (a) $\beta=0$ (BA), (b) $\beta=0.2$, (c) $\beta=0.6$, (d) $\beta=1$ (ER). The four panels show both Forward and Backward continuations, as $\lambda$ is changed in steps of $\delta \lambda=0.01$. The red line is Forward and the yellow line is Backward. The size of the networks is $N=200$ and the average degree is $\langle k\rangle=6$.

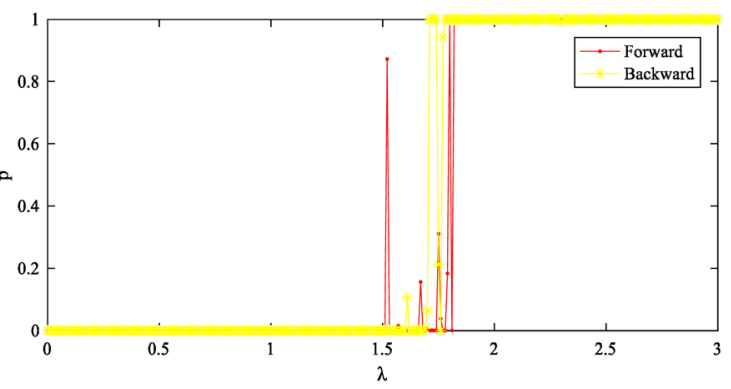

(a)

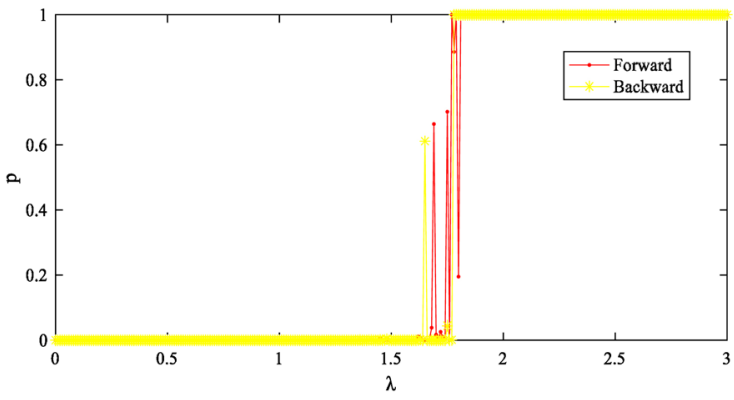

(c)

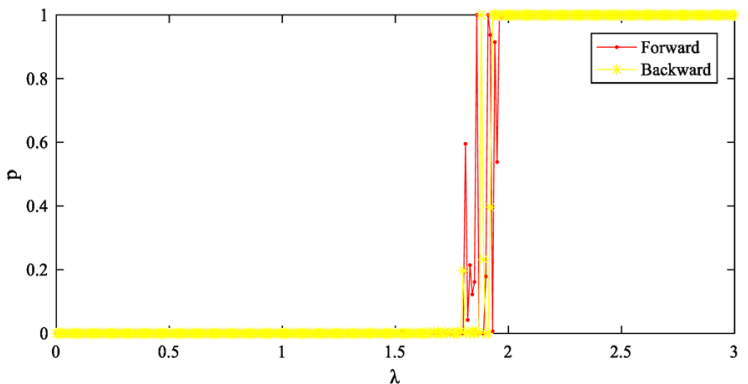

(b)

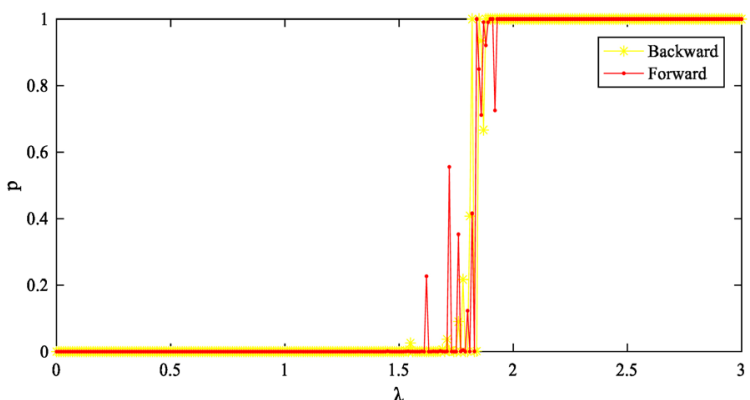

(d)

Figure 2. (color online) The synchronization diagrams $p(\lambda)$ of the networked Lü systems for different networks constructed using the model introduced in [20]. The $\beta$ values in each panel are (a) $\beta=0$ (BA), (b) $\beta=0.2$, (c) $\beta=0.6$, (d) $\beta=1$ (ER). The four panels show both Forward and Backward continuations, as $\lambda$ is changed in steps of $\delta \lambda=0.01$. The red line is Forward and the yellow line is Backward. The size of the networks is $N=200$ and the average degree is $\langle k\rangle=6$. 


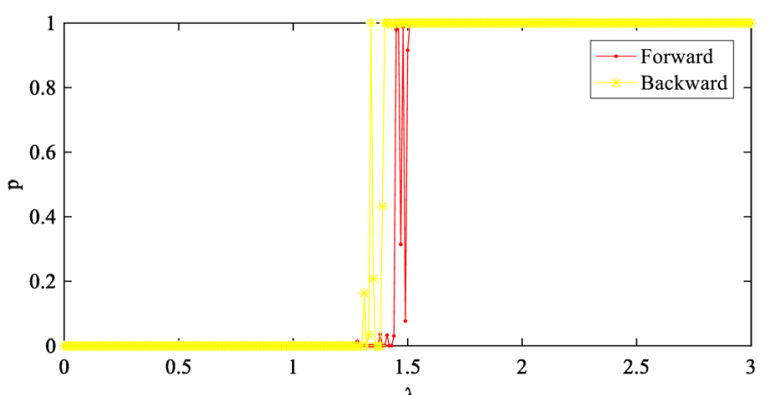

(a)

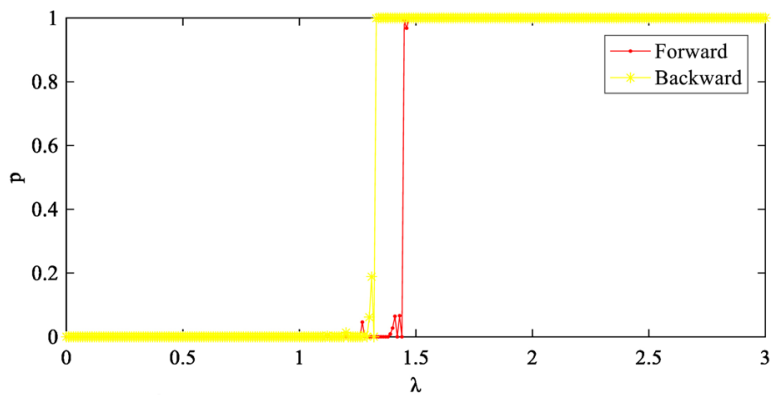

(c)

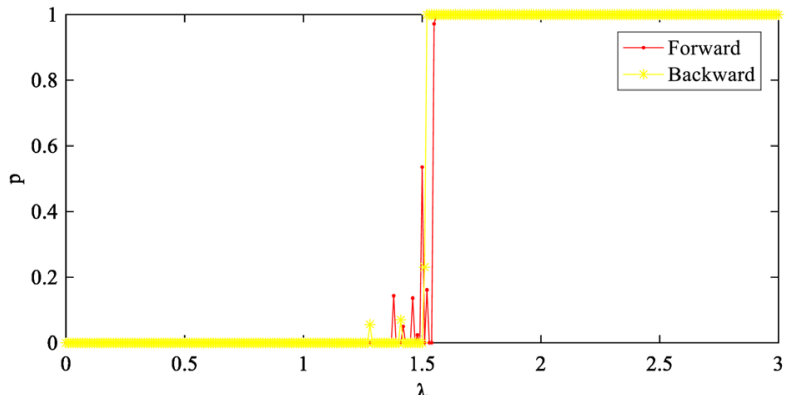

(b)

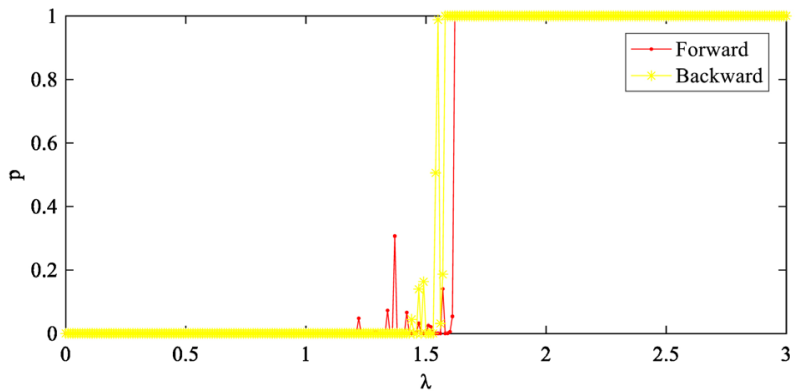

(d)

Figure 3. (color online) The synchronization diagrams $p(\lambda)$ of the networked Chen systems for different networks constructed using the model introduced in [20]. The $\beta$ values in each panel are (a) $\beta=0$ (BA), (b) $\beta=0.2$, (c) $\beta=0.6$, (d) $\beta=1$ (ER). The four panels show both Forward and Backward continuations, as $\lambda$ is changed in steps of $\delta \lambda=0.01$. The red line is Forward and the yellow line is Backward. The size of the networks is $N=200$ and the average degree is $\langle k\rangle=6$.

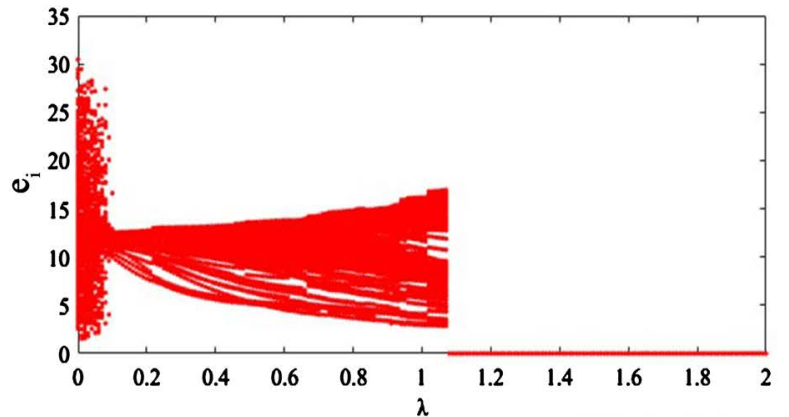

(a)

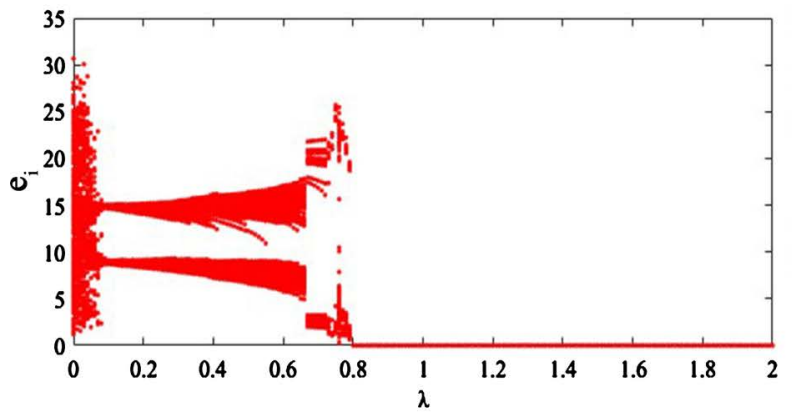

(c)

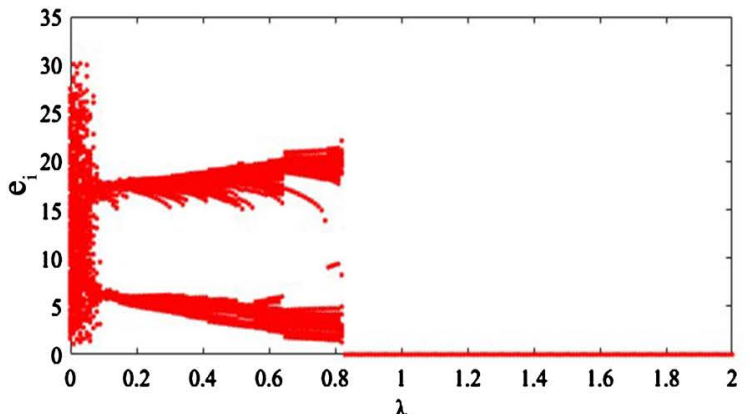

(b)

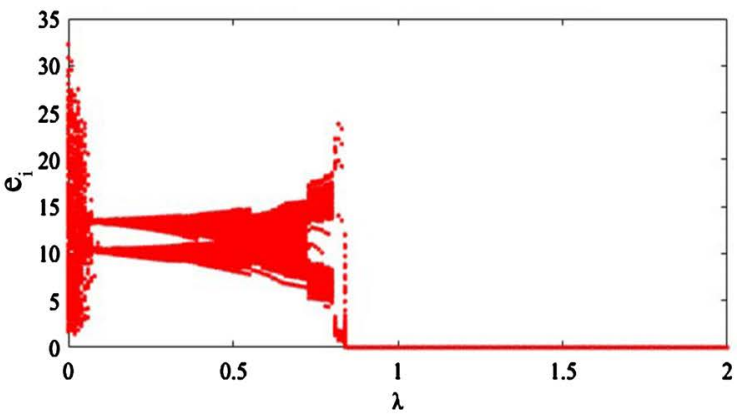

(d)

Figure 4. (color online) The error diagrams $e_{i}(\lambda)$ of the networked Lorenz systems for different networks constructed using the model introduced in [20]. The values in each panel are (a) $\beta=0$ (BA), (b) $\beta=0.2$, (c) $\beta=0.6$, (d) $\beta=1$ (ER). The four panels show errors of all nodes as $\lambda$ is increased in steps of $\delta \lambda=0.01$. The size of the networks is $N=200$ and the average degree is $\langle k\rangle=6$. 


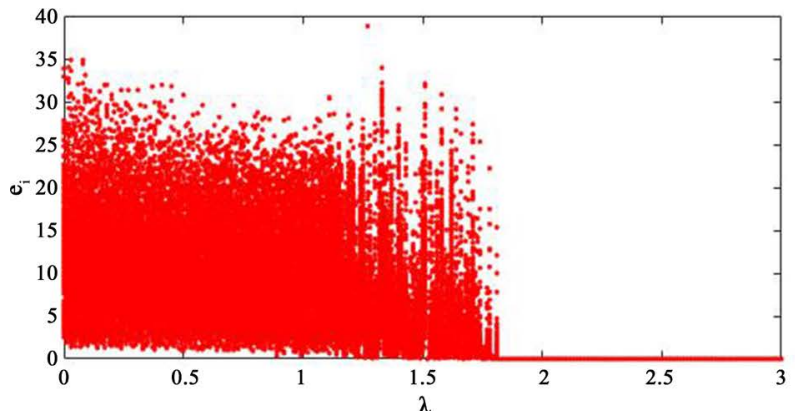

(a)

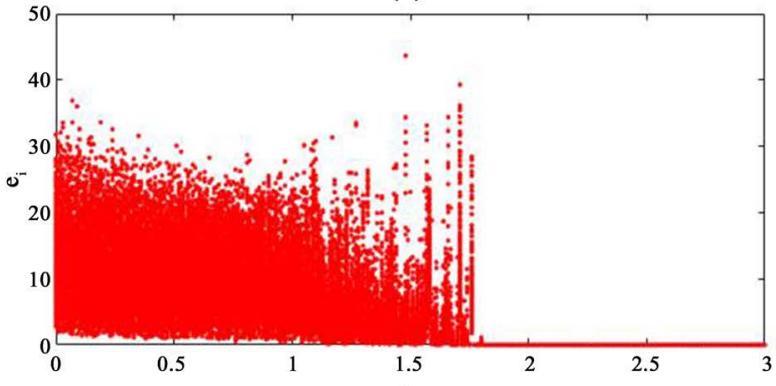

(c)

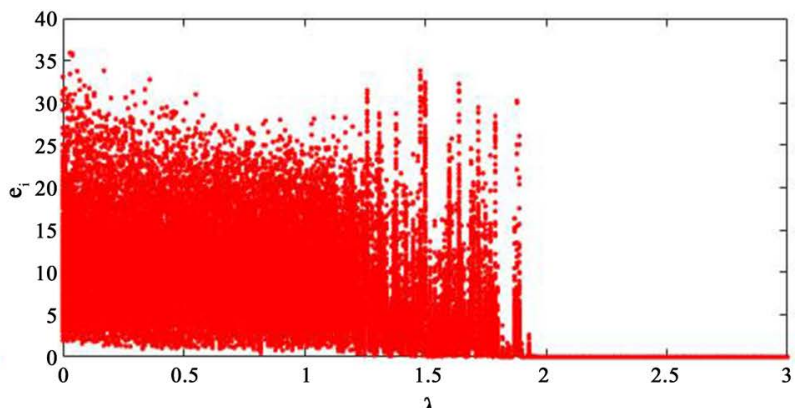

(b)

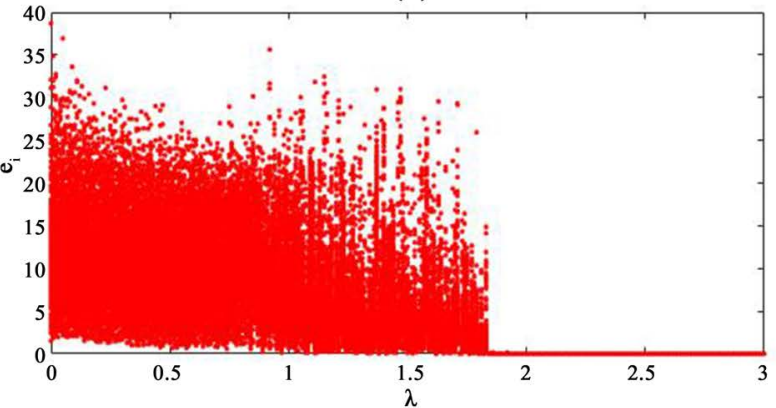

(d)

Figure 5. (color online) The error diagrams $e_{i}(\lambda)$ of the networked Lü systems for different networks constructed using the model introduced in [20]. The $\beta$ values in each panel are (a) $\beta=0$ (BA), (b) $\beta=0.2$, (c) $\beta=0.6$, (d) $\beta=1$ (ER). The four panels show errors of all nodes as $\lambda$ is increased in steps of $\delta \lambda=0.01$. The size of the networks is $N=200$ and the average degree is $\langle k\rangle=6$.

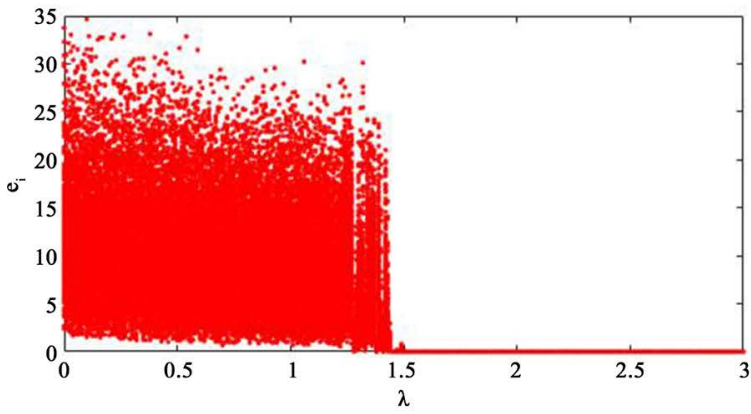

(a)

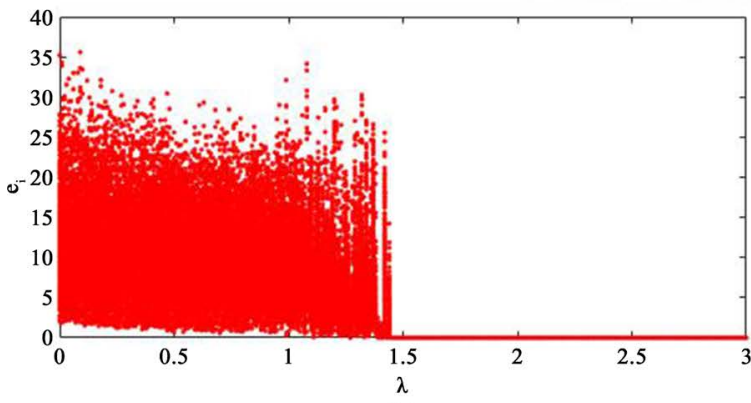

(c)

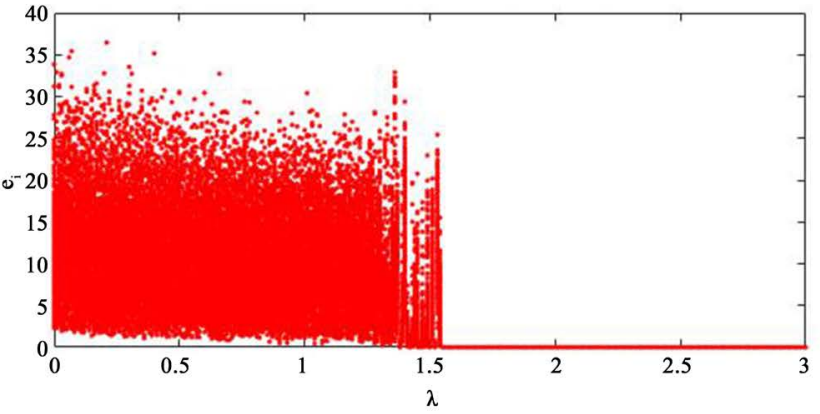

(b)

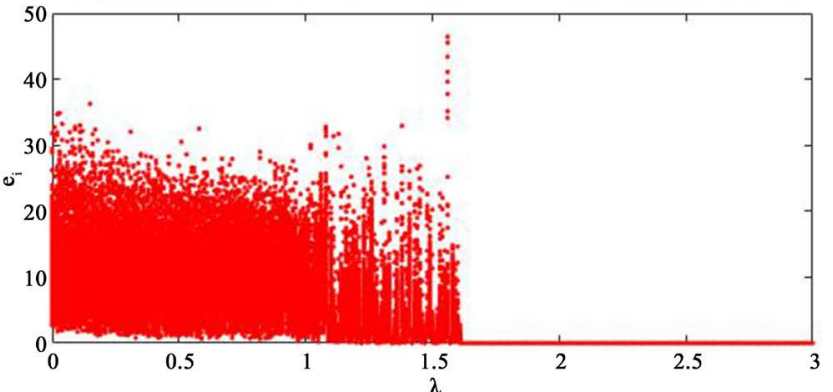

(d)

Figure 6. (color online) The error diagrams $e_{i}(\lambda)$ of the networked Chen systems for different networks constructed using the model introduced in [20]. The $\beta$ values in each panel are (a) $\beta=0$ (BA), (b) $\beta=0.2$, (c) $\beta=0.6$, (d) $\beta=1$ (ER). The four panels show errors of all nodes as $\lambda$ is increased in steps of $\delta \lambda=0.01$. The size of the networks is $N=200$ and the average degree is $\langle k\rangle=6$. 
Thus, the first-order transition of the networked Lorenz systems corresponds to a process in which no microscopic signal of synchronization is observed until the threshold of coupling strength $\lambda_{f}$ is reached.

The conclusive information conveyed by Figure 1 and Figure 4 is that a first-order transition emerging in the networks coupled with Lorenz systems is, indeed, not associated with any specific network structures. It is worth noticing that one can have first-order transitions in coupled Lorenz systems for heterogeneous or homogeneous topologies, without any conditions such as a positive correlation between the graph connections and the node dynamics.

However, the synchronization diagrams of coupled Lü and Chen systems are contrasted with the Lorenz system, between which the differences are very apparent, as shown in Figure $2(\alpha=0.8)$ and Figure $3(\alpha=1)$. There are huge shocks, and even sometimes the coupled systems are suddenly to desynchronize when the networks are almost to synchronize during the Forward continuation. However, the global order parameter $p(\lambda)$ will keep being a stable value, provided the network achieves complete synchronization. This result shows that there exists partial synchronization for coupled Lü and Chen systems. It can be understood as second-order transitions in a certain sense. Moreover, an obvious hysteresis phenomenon from complete synchronization to incoherence is not observed in networks coupled with Lü and Chen systems. Although the red line and the yellow line are not completely coincident, the forward threshold $\lambda_{f}$ is very close to the backward threshold $\lambda_{b}$. We guess that these small distances between $\lambda_{f}$ and $\lambda_{b}$ resulted from systematic errors in numerical simulations. Simultaneously, we can find that the local error parameters of some nodes are gradually reduced to zero, i.e., a second-order transitions towards synchronization take place in networked Lü and Chen systems, as shown in Figure 5 and Figure 6. Finally, we think that explosive synchronization mainly depends on the dynamics of systems based on the above discussions. It may be possible that explosive synchronization emerges by imposing a positive correlation between the heterogeneity of the network structures and node dynamics, which is worthy of further investigation.

\section{The Rössler System as Node Dynamics}

In this section, some numerical simulations of the coupled Rössler systems are presented in different networks. The global synchronization diagrams $p(\lambda)$ and local error diagrams $e_{i}(\lambda)$ are computed, similarly to the coupled unified chaotic systems, as shown in Figure 7 and Figure 8. Numerical simulations show that the coupled Rössler systems achieve synchronization much faster than Lorenz systems for the same network topologies. Therefore, $e_{i}(t)$ becomes zero with a very small $\lambda$.

For each panel in Figure 7 shows a typical second-order transition in Forward process. To analyze deeply the synchronization transition, we monitor the evolution of the dynamical behaviors of every node. Figure 8 shows that the 


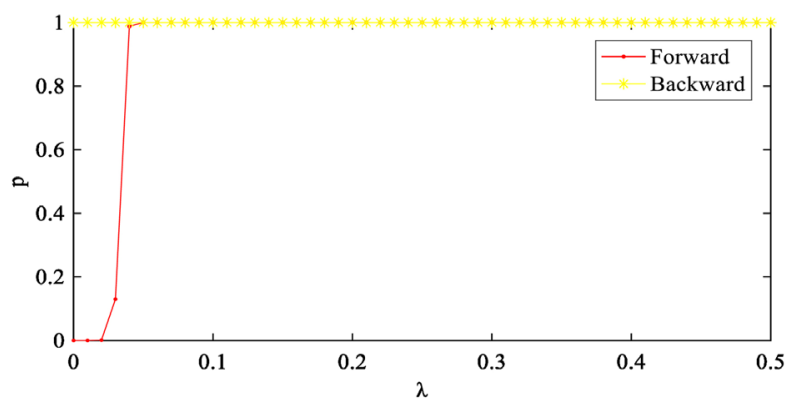

(a)

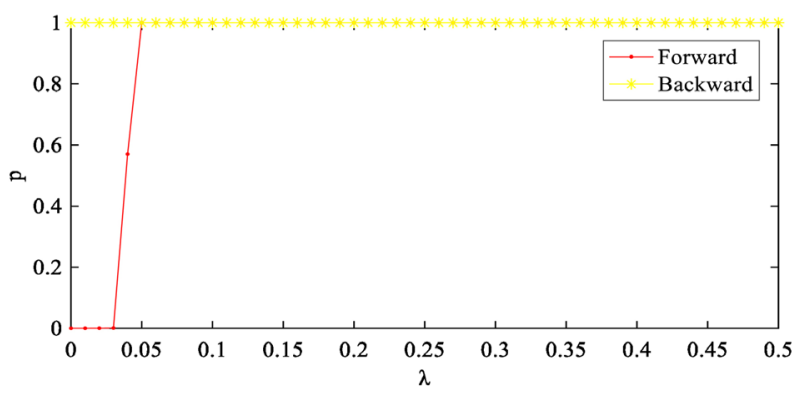

(c)

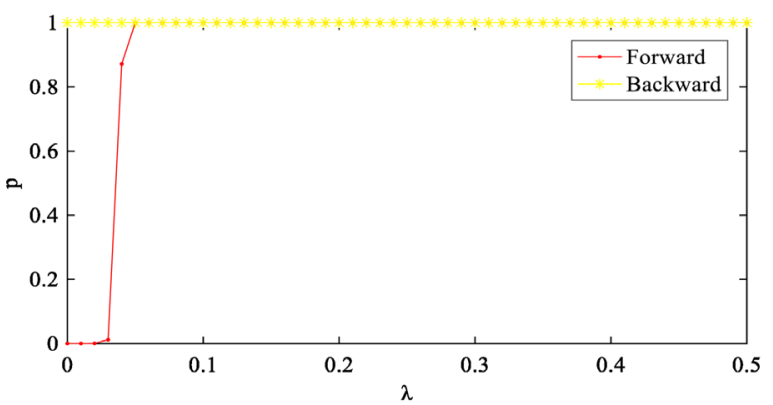

(b)

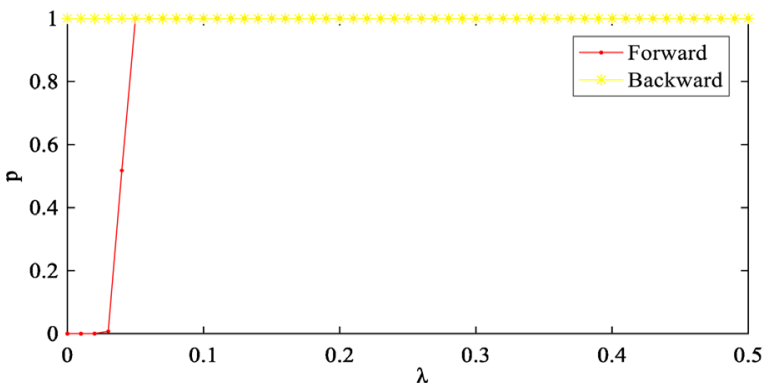

(d)

Figure 7. (color online) The synchronization diagrams $p(\lambda)$ of the networked Rössler systems for different networks constructed using the model introduced in [20]. The $\beta$ values in each panel are (a) $\beta=0$ (BA), (b) $\beta=0.2$, (c) $\beta=0.6$, (d) $\beta=1$ (ER). The four panels show both Forward and Backward continuations as $\lambda$ is changed in steps of $\delta \lambda=0.01$. The red line is Forward and the yellow line is Backward. The size of the networks is $N=200$ and the average degree is $\langle k\rangle=6$.

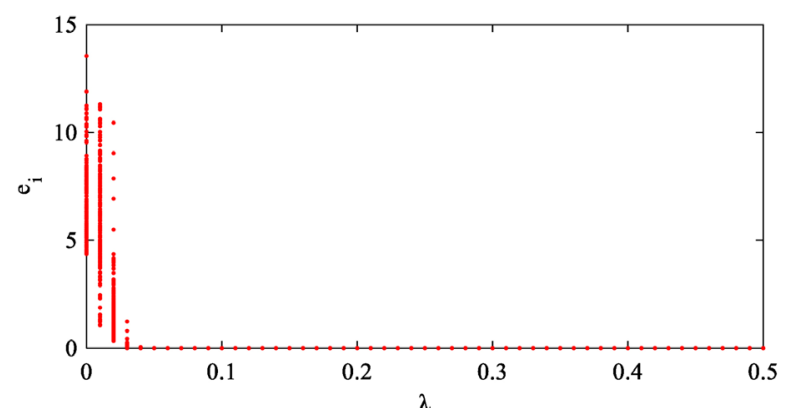

(a)

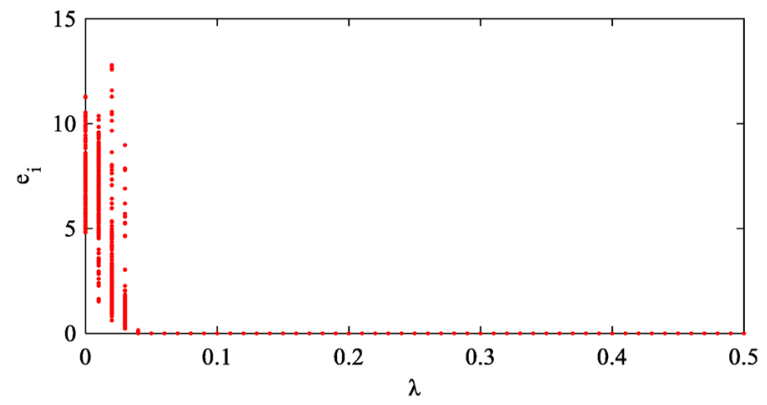

(c)

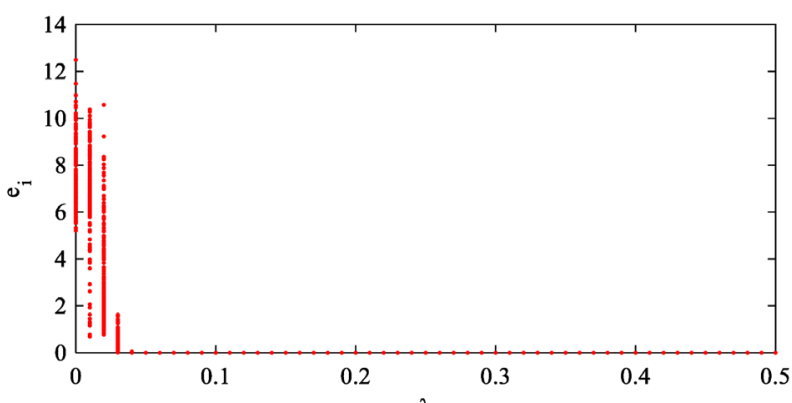

(b)

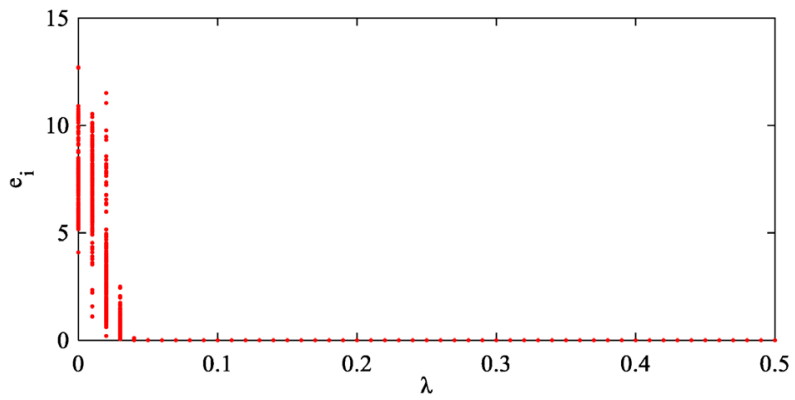

(d)

Figure 8. (color online) The error diagrams $e_{i}(\lambda)$ of the networked Rössler systems for different networks constructed using the model introduced in [20]. The $\beta$ values in each panel are (a) $\beta=0$ (BA), (b) $\beta=0.2$, (c) $\beta=0.6$, (d) $\beta=1$ (ER). The four panels show errors of all nodes as $\lambda$ is increased in steps of $\delta \lambda=0.01$. The size of the networks is $N=200$ and the average degree is $\langle k\rangle=6$. 
local error parameters $e_{i}(\lambda)$ of nodes are continuously reduced to zero, as the coupling strength $\lambda$ is increased, which results in a second-order transition.

Importantly, as shown in Figure 7, in Backward continuations, the coupling constantly decreases, but the network will keep synchronous for all different network structures. We think that the phenomenon is caused by the dynamical features of Rössler systems. To explain it, we plot the Rössler attractors after network synchronization. We find that the trajectory of coupled Rössler systems moves into a periodic orbit once the network synchronizes, as shown in Figure 9. To further verify our conjecture, we plot the $Z$-direction trajectory of one node randomly chosen from the network, as shown in Figure 10. It is clearly observed that the trajectory is periodic. Therefore, synchronization of the coupled Rössler systems will not be destroyed once it is achieved.

\section{Conclusion}

In conclusion, we reported the transitions towards synchronization of coupled chaotic systems in different networks. Our results show that the emergence of explosive synchronization mainly depends on the dynamics of chaotic systems, especially the specific dynamical state at which the chaotic systems. We have given here numerical proof that a first-order and irreversible synchronization transition takes place in a network of coupled Lorenz systems, although the network structures are more homogeneous. It has been seen that the correlation between node dynamics and topological heterogeneity is not a necessary condition for such an explosive transition in networked Lorenz systems. But for the

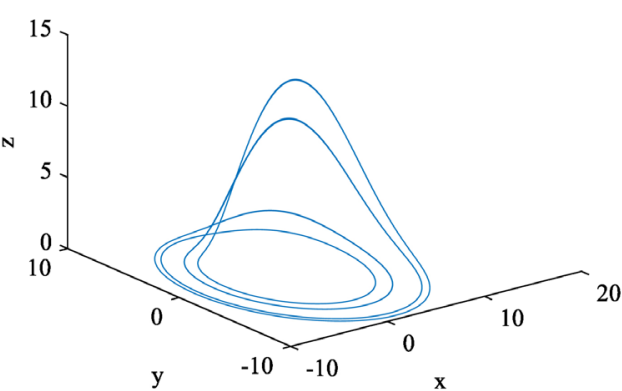

(a)

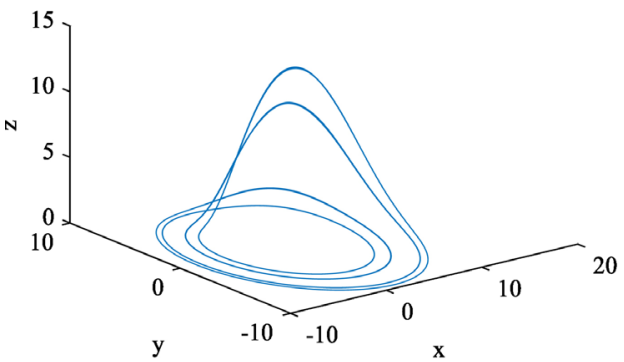

(c)

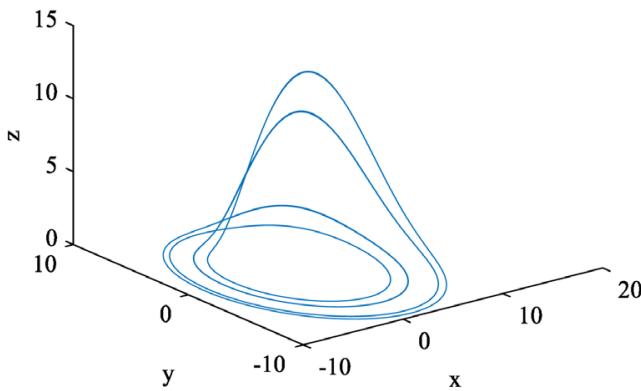

(b)

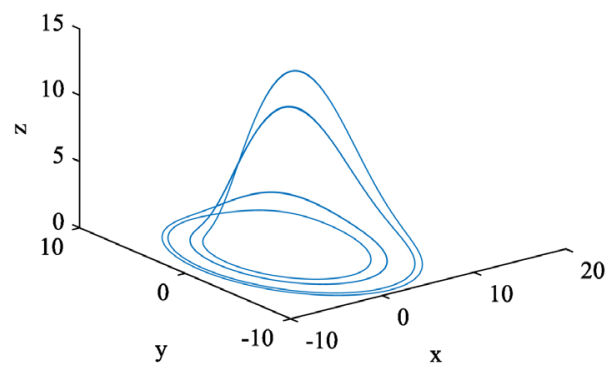

(d)

Figure 9. (color online) The Rössler attractors diagrams for different networks constructed using the model introduced in [20]. The $\beta$ values in each panel are (a) $\beta=0$ (BA), (b) $\beta=0.2$, (c) $\beta=0.6$, (d) $\beta=1$ (ER), and the coupling strength $\lambda=0.25$. 


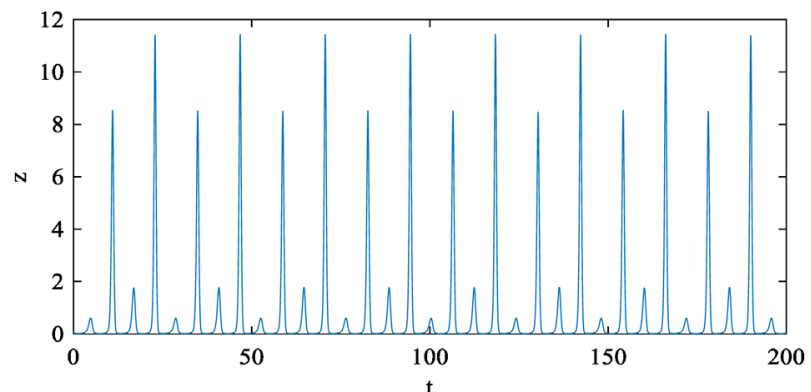

(a)

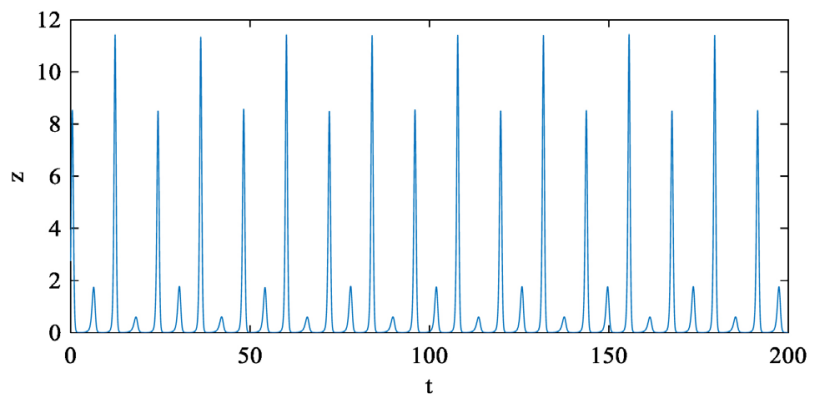

(c)

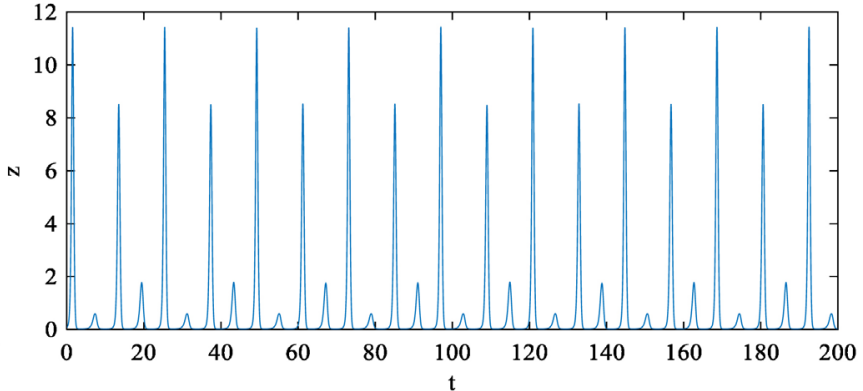

(b)

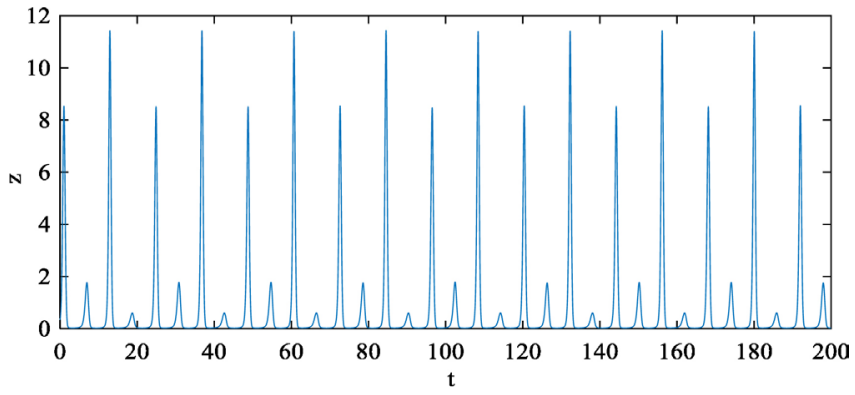

(d)

Figure 10. (color online) $Z$-direction trajectories of one of the Rössler systems after complete synchronization achieved. The $\beta$ values in each panel are (a) $\beta=0$ (BA), (b) $\beta=0.2$, (c) $\beta=0.6$, (d) $\beta=1$ (ER).

networked Lü and Chen systems, a second-order transition towards synchronization is observed, even in the heterogeneous network structures. Surprised, the synchronous state of the networked Rössler systems would not be destroyed once the network achieves complete synchronization, which is because the synchronized Rössler systems move into a periodic orbit. Our findings extend the possibility of encountering first-order transitions to a larger variety of network topologies. This will help us to apply the uncovered mechanism to practice in the future.

\section{Acknowledgements}

This work is supported by the National Natural Science Foundation of China under Grants 61304164 and 61473338, the National Social Science Foundation of China under Grant 18BTJ025, and the Research Project of Hubei Provincial Department of Education under Grant Q20131107.

\section{Conflicts of Interest}

The authors declare no conflicts of interest regarding the publication of this paper.

\section{References}

[1] Watts, D.J. and Strogatz, S.H. (1998) Collective Dynamics of "Small-World" Networks. Nature, 393, 440-442. https://doi.org/10.1038/30918

[2] Newman, M.E.J. and Watts, D.J. (1999) Renormalization Group Analysis of the Small-World Network Model. Physics Letters A, 263, 341-346. 
https://doi.org/10.1016/S0375-9601(99)00757-4

[3] Barabasi, A.L. and Albert, R. (1999) Emergence of Scaling in Random Networks. Science, 286, 509-512. https://science.sciencemag.org/content/286/5439/509 https://doi.org/10.1126/science.286.5439.509

[4] Wu, C.W. (2005) Synchronization in Arrays of Coupled Nonlinear Systems with Delay and Nonreciprocal Time-Varying Coupling. IEEE Transactions on Circuits and Systems II, 52, 282-286. https://doi.org/10.1109/TCSII.2005.846884

[5] Arenas, A., Díaz-Guilera, A., Kurths, J., Moreno, Y. and Zhou, C. (2008) Synchronization in Complex Networks. Physics Reports, 469, 93-153.

https://doi.org/10.1016/j.physrep.2008.09.002

[6] Zhou, J., Lu, J.A. and Lü, J. (2008) Pinning Adaptive Synchronization of a General Complex Dynamical Network. Automatic, 45, 598-599. https://doi.org/10.1016/j.automatica.2007.08.016

[7] Oh, J., Reischmann, E. and Rial, J.A. (2014) Polar Synchronization and the Synchronized Climatic History of Greenland and Antarctica. Quaternary Science Reviews, 83, 129-142. https://doi.org/10.1016/j.quascirev.2013.10.025

[8] He, G., Fang, J.A. and Zhang, W.B. (2016) Synchronization of Switched Complex Dynamical Networks with Non-Synchronized Subnetworks and Stochastic Disturbances. Neurcomputing, 171, 39-47. https://doi.org/10.1016/j.neucom.2015.05.068

[9] Meller, B. and Li, Z. (2017) The Synchronization of Credit Cycles. Journal of Banking \& Finance, 82, 98-111. https://doi.org/10.1016/j.jbankfin.2017.05.011

[10] Sharma, A. (2019) Explosive Synchronization through Dynamical Environment. Physics Letters A, 383, 2051-2055. https://doi.org/10.1016/j.physleta.2019.04.006

[11] Gómez-Gardeñes, J., Gómez, S., Arenas, A. and Moreno, Y. (2011) Explosive Synchronization Transitions in Scale-Free Networks. Physical Review Letters, 106, Article ID: 128701. https://doi.org/10.1103/PhysRevLett.106.128701

[12] Zhao, J.C. (2013) Explosive Synchronization of Complex Networks with Different Chaotic Oscillators. Chinese Physics B, 22, Article ID: 060506.

http://cpb.iphy.ac.cn/EN/10.1088/1674-1056/22/6/060506 https://doi.org/10.1088/1674-1056/22/6/060506

[13] Zhang, X.Y., Boccaletti, S., Guan, S.G. and Liu, Z.H. (2015) Explosive Synchronization in Adaptive and Multilayer Networks. Physical Review Letters, 114, Article ID: 038701. https://doi.org/10.1103/PhysRevLett.114.038701

[14] Boccaletti, S., Almendral, J.A., Guan, S., Leyva, I., Liu, Z., Sendiña-Nadal, I., Wang, Z. and Zou, Y. (2016) Explosive Transitions in Complex Networks' Structure and Dynamics: Percolation and Synchronization. Physics Reports, 660, 1-94. https://doi.org/10.1016/j.physrep.2016.10.004

[15] Huang, C.H. and Cao, J.D. (2017) Active Control Strategy for Synchronization and Anti-Synchronization of a Fractional Chaotic Financial System. Physica A: Statistical Mechanics and Its Applications, 473, 262-275. https://doi.org/10.1016/j.physa.2017.01.009

[16] Gao, Y.C., Fu, C.J., Cai, S.M., Yang, C. and Stanley, H.E. (2019) Repulsive Synchronization in Complex Networks. Chaos: An Interdisciplinary Journal of Nonlinear Science, 29, Article ID: 053130. https://doi.org/10.1063/1.5089567

[17] Cao, L., Tian, C.H., Wang, Z.H., Zhang, X.Y. and Liu, Z.H. (2018) Influence of Stochastic Perturbations on the Cluster Explosive Synchronization of Second-Order Kuramoto Oscillators on Networks. Physical Review E, 97, Article ID: 022220. https://doi.org/10.1103/PhysRevE.97.022220 
[18] Lu, J.H., Chen, G.R., Cheng, D.Z. and Celikovský, S. (2002) Bridge the Gap between the Lorenz System and the Chen System. International Journal of Bifurcation and Chaos, 12, 2917-2926. https://doi.org/10.1142/S021812740200631X

[19] Rössler, O.E. (1976) An Equation for Continuous Chaos. Physics Letter A, 57, 397-398. https://doi.org/10.1016/0375-9601(76)90101-8

[20] Gómez-Gardeñes, J. and Moreno, Y. (2006) From Scale-Free to Erdos-Rényi Networks. Physical Review E, 73, Article ID: 056124. https://doi.org/10.1103/PhysRevE.73.056124

[21] Li, X. and Chen, G.R. (2003) Synchronization and Desynchronization of Complex Dynamical Networks: An Engineering Viewpoint. IEEE Transactions on Circuits and Systems I, 50, 1381-1390. https://doi.org/10.1109/TCSI.2003.818611 